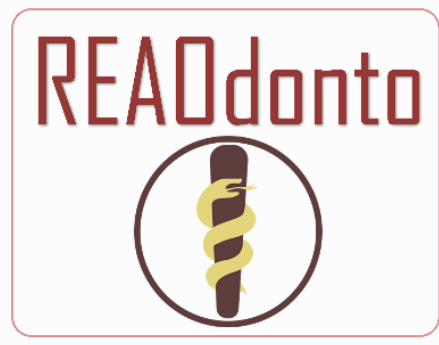

Revista Eletrônica Acervo
Odontológico

\section{ARTIGO ORIGINAL}

Recebido em: 5/2020

Aceito em: 6/2020

Publicado em: 9/2020

\title{
Associação entre a odontologia estética e autoestima
}

\author{
Association between aesthetic dentistry and self-esteem
}

Asociación entre odontología estética y autoestima

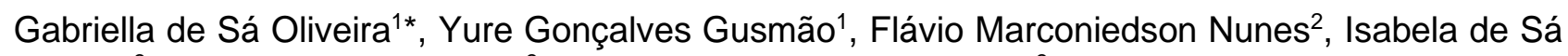
Oliveira $^{3}$, Lara Santos Cangussu ${ }^{3}$, Marcelo Cavalcanti Gonçalves ${ }^{3}$.

\begin{abstract}
Resumo: Este artigo teve como objetivo realizar análise sobre a associação entre o trabalho da odontologia estética e a autoestima dos pacientes. Para isso foi realizado estudo bibliométrico de publicações científicas sobre o assunto. A busca inicial das referências bibliográficas foi efetuada na Biblioteca Virtual de Saúde (BVS), de forma integrada, de maio a agosto de 2019. O estudo contou com a seleção final de 20 referências de 212 publicadas a partir do ano de 2007. Dessas 212 referências, 192 foram excluídas por não atenderem os critérios de inclusão propostos no protocolo da pesquisa. De acordo com as referências, os fatores encontrados que impactam diretamente na autoestima dos indivíduos foram o edentulismo, amelogênese imperfeita, fluorose, dentes anteriores fraturados, diastema, sorriso gengival e posição inadequada da mandíbula. A deformidade dentofacial influencia não somente na autoconfiança, como também nos relacionamentos e qualidade de vida, resultando em impactos psicossociais. Todas as referências utilizadas neste estudo corroboram que a odontologia estética além de impactar no reestabelecimento da estética e função, atua na recuperação da autoestima. Desse modo, é de suma importância a preparação do profissional para que ele esteja apto a atender essa necessidade.
\end{abstract}

Palavras-chave: Odontologia estética, Autoestima, Qualidade de vida.

\begin{abstract}
This article aimed to analyze the association between the work of cosmetic dentistry and patients' self-esteem. For this, a bibliometric study of scientific publications on the subject was carried out. The initial search for bibliographic references was carried out in the Virtual Health Library (BVS), in an integrated manner, from May to August 2019. The study included the final selection of 20 references from 212 published from the year 2007. Of these 212 references, 192 were excluded because they did not meet the inclusion criteria proposed in the research protocol. According to the references, the factors found that directly impact individuals self-esteem were edentulism, imperfect amelogenesis, fluorosis, fractured anterior teeth, diastema, gengival smile and inadequate jaw position. Dentofacial deformity influences not only selfconfidence, but also relation ships and quality of life, resulting in psychosocial impacts. All the references used in this study corroborate that aesthetic dentistry, in addition to impacting the reestablishment of aesthetics and function, acts in the recovery of self-esteem. Thus, it is of utmost importance to prepare the professional so that he is able to meet this need.
\end{abstract}

Keywords: Cosmetic dentistry, Self-esteem, Quality of life.

Resumen: Este artículo tuvo como objetivo analizar la asociación entre el trabajo de la odontología cosmética y la autoestima de los pacientes. Para ello, se realizó un estudio bibliométrico de publicaciones

${ }^{1}$ Faculdades Integradas do Norte de Minas (FUNORTE), Montes Claros - MG.

*E-mail: gabrielladesapsicologia@gmail.com

${ }^{2}$ Centro Universitário (UNIFIPMOC), Montes Claros - MG.

${ }^{3}$ Universidade Estadual de Montes Claros (UNIMONTES), Montes Claros - MG. 
científicas sobre el tema. La búsqueda inicial de referencias bibliográficas se llevó a cabo en la Biblioteca Virtual en Salud (BVS), de manera integrada, de mayo a agosto de 2019. El estudio incluyó la selección final de 20 referencias de 212 publicadas desde el año 2007. De estas 212 Se excluyeron 192 referencias porque no cumplían los criterios de inclusión propuestos en el protocolo de investigación. Según las referencias, los factores que afectan directamente la autoestima de los individuos fueron edentulismo, amelogénesis imperfecta, fluorosis, dientes anteriores fracturados, diastema, sonrisa gingival y posición inadecuada de la mandíbula. La deformidad dentofacial influye no solo en la autoconfianza, sino también en las relaciones y la calidad de vida, lo que resulta en impactos psicosociales. Todas las referencias utilizadas en este estudio corroboran que la odontología estética, además de impactar el restablecimiento de la estética y la función, actúa en la recuperación de la autoestima. Por lo tanto, es de suma importancia preparar al profesional para que pueda satisfacer esta necesidad.

Palabras clave: Odontología cosmética, Autoestima, Calidad de vida.

\section{INTRODUÇÃO}

A aparência do indivíduo é um elemento fundamental para fazer com que ele se sinta bem fisicamente e emocionalmente, além de motivado para os desafios cotidianos. E, para a Odontologia, é fundamental considerar todos os aspectos funcionais, estéticos e psicológicos, pois todos estão diretamente associados com a saúde geral do indivíduo (BARRETO JO, et al., 2019).

A face possui lugar de destaque e é nela onde está inserido o sorriso. Desse modo, a deformidade dentofacial possui potencial psicológico e social destrutivo, uma vez que, exibe reflexos negativos nas interações sociais, influenciando não somente na autoconfiança dos pacientes, mas também nos relacionamentos externos e qualidade de vida, resultando em desvantagens sociais e psicológicas. A percepção estética é variável, sendo de responsabilidade profissional percebê-las e alertar para o significado estético de cada diferença (GALLÃO S, et al., 2009).

Segundo Nicodemo D, et al. (2007), a estética relacionada à face pode ser oriunda de diversas implicações clínicas como amelogênese imperfeita, hiperplasias, má oclusão, dentes supranumerários, anodontia, agenesia, perdas dentárias, lábio leporino, assimetrias faciais, fraturas e sequelas, lesões tumorais, discrepâncias maxilomandibulares, entre outros, bem como inúmeras formas de tratamento. Essas deformidades dentofaciais são também psicossociais e estas podem manifestar o anseio em resolver suas questões pessoais e sociais com a mudança física, ou seja, com a melhora de sua fisionomia pela correção terapêutica.

Ainda de acordo com Nicodemo D, et al. (2007), os aspectos psicossociais estão diretamente relacionados ao tratamento odontológico, pois a percepção estética facial induz a formação da imagem corporal, da identidade e da auto estima. Logo, o homem sendo um ser que consegue expressar sentimentos por meio da face, em especial, pelo sorriso, os aspectos estéticos negativos podem afetá-lo, principalmente quanto ao convívio social e psicológico em decorrência de uma autoestima abalada. (OLIVEIRA D, 2019).

Ademais, de acordo com suas condições físicas e emocionais, o individuo pode sentir que não está de acordo com o ideal estético, se retraindo e descrevendo sinais e sintomas de isolamento e depressão. (FAIS LMG, 2007). Conforme Barreto JO (2019) e Pedron IG (2014) os profissionais de saúde devem se preocupar não só com o bem-estar físico dos pacientes, mas também com o bem-estar mental garantindoIhes qualidade de vida, pois, assim como os procedimentos médicos, os odontológicos devem garantir além da promoção de saúde, a estética facial proporcionando uma melhor socialização e expressão dos seus sentimentos.

Sendo assim, a odontologia em seu processo de reparação estética é essencial também para a recuperação dos aspectos psicossociais. Desta forma, o objetivo deste trabalho foi realizar uma revisão de literatura a respeito da associação da odontologia estética para a reabilitação do paciente como um todo, restabelecendo sua autoestima e impactando diretamente na qualidade de vida. 


\section{MÉTODOS}

O presente trabalho trata-se de um estudo bibliométrico de publicações científicas sobre a associação entre a odontologia estética e autoestima de pacientes brasileiros. A busca inicial das referências bibliográficas foi efetuada na Biblioteca Virtual de Saúde (BVS), de forma integrada, em agosto de 2019, no qual foram avaliados artigos publicados entre os anos de 2007 e 2020. Os descritores utilizados foram: Autoestima e Odontologia.

Os critérios de inclusão definidos na pesquisa foram para artigos que relacionassem os impactos da odontologia estética e a autoestima em pacientes brasileiros, que abrangessem todos os tipos de intervenções (cirúrgica e não cirúrgica), que tivessem resumos disponíveis na íntegra, que possuíssem apresentação de evidências científicas fortes, apresentação de casos clínicos finalizados e estivessem em português. Já os critérios de exclusão foram para artigos que não demonstrassem relação entre esses dois termos e que não tratassem sobre pacientes brasileiros.

Efetuou-se a seleção pelos títulos e resumos e depois pelo texto na íntegra. Neste estudo serão apresentadas informações sobre: ano, idioma, periódico de publicação e tema central discutido no artigo.

\section{RESULTADOS}

O estudo contou com a seleção final de 20 referências de 212 publicadas a partir do ano de 2007. Dessas 212 referências, 192 foram excluídas por não atenderem os critérios de inclusão propostos no protocolo da pesquisa. Entre os 20 artigos científicos selecionados 3 foram publicados em 2007, 3 em 2009, 1 em 2010, 1 em 2011, 1 em 2012, 2 em 2014, 1 em 2016, 2 em 2017, 1 em 2018, 3 em 2019 e 2 em 2020, como descrito na Tabela 1.

Os artigos foram publicados em periódicos nacionais e internacionais: Rev. Fac. Odont $(n=1)$, Revista Odontológica de Araçatuba $(n=6)$, Arco. Health. Invest $(n=4)$, Rev. Dental Press Ortodon Ortop Facial $(n=2)$, Rev. Inst Ciência. Saúde ( $n=1)$, Clínica-Internacional Journal of Brazilian Dentistry $(n=1)$, Rev Dental Press Estet ( $n=2)$, Rev. Odontol. Univ. Cid. São Paulo $(n=1)$ e Rev. Gaúcha Odontol $(n=2)$.

Diferentes formas de associação entre odontologia estética e autoestima foram apresentadas nos artigos. Pode-se destacar o procedimento de "Laminados Cerâmicos", discutido em 05 artigos, conforme descrito na Tabela 2.

Nessa perspectiva, o trabalho de Oliveira et al. (2018), relatou a utilização de laminados cerâmicos ultrafinos em um caso de amelogênese imperfeita com o objetivo de restabelecer a estética e função. Após o acompanhamento de um ano, os autores puderam concluir que os laminados cerâmicos são uma alternativa interessante para o tratamento definitivo de casos mais brandos de amelogênese imperfeita, com otimização dos resultados estéticos e recuperação da autoestima do paciente.

Já o procedimento "Cirurgia ortognática" foi trabalhado em 3 artigos, conforme descrito na Tabela 2. Desse modo, Ferreira, et al. (2007) demonstraram em seu estudo que o segundo principal motivo pelo o qual os pacientes procuram pela correção cirúrgica era a melhoria da estética. Desse modo, ele chamou atenção de como a deformidade facial causa impactos negativos, influenciando não somente na autoconfiança, mas também em seus relacionamentos externos, conforme descrito na Tabela 2.

Outras intervenções que interferem diretamente na autoestima dos pacientes também foram discutidos, como "Reabilitação com próteses" $(n=3)$; "Intervenção ortodôntica" $(n=1)$; "Utilização de toxina botulínica" $(n=3)$; "Utilização de resina" ( $n=3)$; "Utilização de clareamento dental" ( $n=1)$; e "Avaliação" $(n=1)$, conforme descrição na Tabela 2. 
Tabela 1- Descrição quantitativa dos artigos conforme ano de publicação.

\begin{tabular}{ccc}
\hline Ano de publicação & N & $\%$ \\
\hline 2007 & 3 & 15 \\
2009 & 3 & 15 \\
2010 & 1 & 5 \\
2011 & 1 & 5 \\
2012 & 1 & 5 \\
2014 & 2 & 10 \\
2016 & 1 & 5 \\
2017 & 2 & 10 \\
2018 & 1 & 5 \\
2019 & 3 & 15 \\
2020 & 2 & 10 \\
\hline Total & 20 & 100
\end{tabular}

Fonte: Oliveira GS, et al., 2020.

Tabela 2 - Descrição quantitativa dos procedimentos discutidos nos artigos científicos.

\begin{tabular}{cll}
\hline Assuntos centrais discutidos nos artigos & N & (\%) \\
\hline Reabilitação com próteses & 3 & 15 \\
Restauração com laminados cerâmicos & 5 & 25 \\
Intervenção Ortodôntica & 1 & 5 \\
Cirurgia Ortognática & 3 & 15 \\
Utilização de botox & 3 & 15 \\
Utilização de resina & 3 & 15 \\
Clareamento & 1 & 5 \\
Avaliação & 1 & 5 \\
\hline Total & 20 & 100
\end{tabular}

Fonte: Oliveira GS, et al., 2020.

\section{DISCUSSÃO}

\section{Características das Principais alterações odontológicas que afetam a autoestima}

Como demonstrado nos resultados, diversos estudos apresentam a relação entre odontologia, estética e autoestima, garantindo o retorno do funcionamento dental e a estética desejada que eleva a autoestima do individuo. Por outra vertente, é possível relacionar os processos que acometem a integridade estrutural e funcional dos dentes, causando diversos problemas graves locais ou ate mesmo sistêmicos.

As alterações mais recorrentes que agravam a estética ou função dos dentes estão relacionadas com os processos patológicos orais e ou más formações ligadas à estrutura, alteração do aspecto ou traumas. Assim a importância de conhecer os processos e agentes que acometem os dentes é de suma relevância para um tratamento adequado, pois consequente os impactos gerados podem acarretar problemas que afetam a autoestima do individuo, ou o surgimento de outros agravantes (BENEDITO FCS, et al., 2020).

Existem vários fatores que ajudam na modificação da cor natural dos dentes. Batista SHB, et al. (2009) relatam que as alterações na amelogênese que aparecem com maior frequência na literatura odontológica são as hipoplasias, a opacidade demarcada e difusa e a amelogênese imperfeita, ligando-se a alterações na estética dental.

A hipoplasia é uma alteração associada ao desenvolvimento do esmalte sendo de origem hereditária ou por consequência de eventos sistêmicos ou locais durante o desenvolvimento dos dentes. Pode ser compreendida pela redução da espessura, resultando na aparência opaca ou translúcida do dente, ocorrem na forma de fosseta ou de sulco, ambos de forma única ou múltipla apresentando ausência parcial ou completa de esmalte dentário sobre uma área considerável de dentina (KRAMER PF, et al., 2009). 
No estudo de Souza AB, et al. (2010) clinicamente foram observados estruturas hipoplásicas manchas brancas e incisuras nos dentes anteriores de indivíduos jovens do sexo feminino, relatando o desconforto e os problemas gerados pelas questões estéticas.

Alterações ligadas às modificações do aspecto dental são muito recorrentes. A opacidade demarcada e opacidade difusa representam modificações que levam a alterações do esmalte dental em superfície lisa ou profunda, modificando o pigmento natural e alterando as margens as deixando irregulares e pontiagudas, podem representar graus severos e irreversíveis no local afetado (CORREIA SF, et al., 2007).

Oliveira D, et al. (2018), realizaram estudo sobre a amelogênese imperfeita, grupo de condições com alterações no desenvolvimento na estrutura do esmalte dentário que afetam a dentição decídua e permanente com implicações relacionadas à estética prejudicada, hipersensibilidade dentária, dificuldade na higiene bucal, cáries recorrentes, inflamação gengival e perda da dimensão vertical.

Outro fator de grande importância está relacionado com a agenesia dental que faz parte do grupo de anomalias dentárias complexas, consistindo na ausência congênita de dentes, podendo estar associada a uma síndrome genética ou aparecer como um caso isolado. Assim a agenesia dental demonstra um impacto na função dos dentes, além de causar problemas psicossociais no individuo, baseados de limitações e interferências estéticas que agravam a terapêutica ortodôntica (DE CASTRO JUNIOR FM, 2019).

Dentre as doenças em destaque na alteração funcional e estética dental, a doença periodontal é a mais recorrente, agravando tecidos periodontal de suporte, tendo ação de microrganismos e seus fatores de virulência, podendo levar a complicações graves ou ate a morte (SOUZA AB, et al., 2010).

De acordo com Ferreira MC, et al. (2017) que em estudo avaliou os impactos da doença periodontal na qualidade de vida de adolescentes, adultos e idosos, a doença periodontal estava associada a um impacto negativo na qualidade de vida, com periodontite severa que exerce o impacto mais significativo, comprometendo aspectos relacionados à função e estética.

Segundo Ferreira MC, et al. (2017) que em estudo avaliou os impactos da doença periodontal na qualidade de vida de adolescentes, adultos e idosos, a doença periodontal estava associada a um impacto negativo na qualidade de vida, com periodontite severa que exerce o impacto mais significativo, comprometendo aspectos relacionados à função e estética.

A cárie por sua vez apresenta-se como uma doença crônica e multifatorial, tendo característica clínica apresentando manchas brancas e opacas no esmalte dental, essas manchas ocorrem devido à desmineralização pela presença do biofilme dental. A evolução desta fase consiste no surgimento de cavidades pela perda da estrutura dental que, se não paralisado, pode trazer a destruição de todo o dente associado a processos infecciosos ou até mesmo sua perda, resultando em complicações sistêmicas e psicossociais (LUNARDELLI SE, et al., 2016).

Deve-se destacar a presença dos traumatismos dentro-alveolares como formas atípicas da alteração da estrutura dental estética funcional, que são originados por vários fatores, tais como, acidentes de trânsito, acidentes domésticos, acidentes de trabalho, agressões dentre outros. Dependendo do tipo de traumatismo e região os dentes podem apresentar perda de parte da estrutura periodontal de proteção e suporte, fraturas coronárias e/ou radiculares, mineralizações pulpares, necrose pulpar, reabsorções internas e externas das raízes até a perda dental (PINO YC, et al., 2020).

\section{Odontologia estética relacionada a aspectos psicológicos comportamentais e de autoestima}

De acordo com Cardoso CAB, et al. (2011), comprometimentos dentários podem induzir alterações comportamentais, de ajuste social e de qualidade de vida, aspectos estes que devem ser considerados no planejamento das intervenções. Nicodemo D, et al. (2007) ressaltaram que pacientes com deformidades dentofaciais podem apresentar dificuldades com a mastigação e a fala, desordens temporomandibulares, preocupação com a imagem corporal e baixa autoestima. Comumente, a busca pelo tratamento ortocirúrgico é motivada para melhorar os aspectos estéticos, funcionais e psicossociais. 
Os autores Marcondes R, et al. (2012) e Bezerra RB, et al. (2014), em consonância, afirmam que o sorriso nunca teve um papel tão importante na vida do ser humano como atualmente, devendo os cirurgiões-dentistas atentarem-se a esses detalhes durante a avaliação e agirem em favor de uma resolução real e duradoura de problemas relacionados à aceitação e autoestima.

Oliveira D (2019), realizaram estudo sobre a amelogênese imperfeita. Esta patologia dentária, segundo os autores, é capaz de provocar sentimentos de constrangimento e exclusão, baixa autoestima e consequências na qualidade de vida. Ainda segundo o autor, após o tratamento com laminados cerâmicos, é possível identificar elevadas taxas de satisfação, com consequente recuperação da autoestima. Vieira AC, et al. (2018), corroborando com os tratamentos com laminados cerâmicos, afirmaram que para obter maior êxito e durabilidade do tratamento restaurador é necessário combinar o sucesso da técnica ao diagnóstico preciso e planejamento individualizado de cada caso.

De acordo com Nicodemo D, et al. (2007) pacientes procuram a correção cirúrgica motivados a melhorar o aspecto funcional e a estética, com fantasias relacionadas a aperfeiçoamento das relações sociais e a aparência. Isso significa que os aspectos psicossociais estão diretamente ligados ao tratamento, constatando que a aparência facial influencia a imagem corporal, identidade e autoestima.

Já a autora Tiveron AHB (2014) concluiu, em relação ao sorriso gengival, que para alguns pacientes, essa aparência estética não afeta nenhuma de suas atividades, porém para outros, o incômodo é enorme, sendo necessário realizar intervenções cirúrgicas para diminuir os efeitos estéticos e psicológicos. Ainda segundo a autora, atualmente observa-se, na odontologia, uma procura cada vez maior por procedimentos estéticos em virtude de o indivíduo estar inserido numa sociedade onde a aparência tem grande importância na sua aceitação e autoestima. Diferenças emocionais em pacientes que necessitam do uso de próteses dentárias também foram observadas por Barreto JO, et al. (2019). Portanto, é importante ressaltar que, como afirma Bezerra RB, et al. (2014), o sorriso estimula o cérebro a liberar endorfina e serotonina, substâncias responsáveis pela sensação de prazer e felicidade, além de ativarem o sistema imunológico, colaborando para a prevenção de doenças causadas pelo estresse.

Quanto os tratamentos realizados na infância, Cardoso CAB, et al. (2011), realizou estudo sobre a reabilitação bucal na primeira infância e afirmou que o ingresso da criança no ambiente escolar e sua socialização atualmente acontecem mais cedo, em torno de 2 a 3 anos de idade, e não mais aos 6 anos, quando é normal o convívio entre os colegas com falhas dentárias transitórias. Com isso, a criança com perda dentária prematura, torna-se alvo de brincadeiras e ridicularização entre os amigos. A autora concluiu que o tratamento na infância resulta na eliminação da dor, recuperação da função e da estética, o que impacta diretamente no aspecto psicológico infantil, além de alteração na conduta da criança e dos pais frente à saúde bucal.

Todas as referências utilizadas neste estudo corroboram que a odontologia estética, além de impactar no reestabelecimento da estética e função, atua na recuperação da autoestima.

\section{CONSIDERAÇÕES FINAIS}

Consideramos que este estudo contribui para as ações dos profissionais envolvidos no atendimento de pacientes em processo de reabilitação dentofacial e que tenham perturbações relacionadas à autoestima $e$ autoimagem, promovendo melhor qualidade de vida. Conclui-se que a deformidade dentofacial possui potencial psicológico e social destrutivo, visto que interfere interações sociais, influenciando não somente na autoconfiança dos pacientes, mas também nos relacionamentos externos e qualidade de vida, resultando em comprometimentos sociais e psicológicos. Ademais, neste estudo, constatou-se que as alterações mais recorrentes que agravam a estética ou função dos dentes estão relacionadas com os processos patológicos orais e ou más formações ligadas à estrutura, alteração do aspecto ou traumas. Por fim, houve consonância entre autores no qual afirmaram que o sorriso nunca teve um papel tão importante na vida do ser humano como tem atualmente, devendo os cirurgiões dentistas agir em favor de uma resolução real e duradoura de problemas relacionados à aceitação e autoestima.

REAOdonto | Vol. 1 | e3892 | DOI: https://doi.org/10.25248/REAOdonto.e3892.2020 Página 6 de 7 


\section{REFERÊNCIAS}

1. BARRETO JO, et al. Impactos psicossociais da estética dentária na qualidade de vida de pacientes submetidos a próteses: revisão de literatura. Arch Health Invest, 2019; 8(1):48-52.

2. BATISTA AMR, et al. Alterações na amelogênese e suas implicações clínicas: relatos de casos. Revista da Faculdade de Odontologia de Porto Alegre, 2009; 50(1): 9-15.

3. BENEDITO FCS, et al. Saúde bucal de universitários internacionais: da importância ao conhecimento e condutas frente às patologias orais. Revista de Pesquisa: Cuidado é Fundamental, 2020; 355-361.

4. BEZERRA RB, et al. Reabilitação estética e funcional do sorriso: relato de caso clínico. Revista Odontológica de Araçatuba, 2014; 35(1): 34-37.

5. CARDOSO CAB, et al. Reabilitação bucal na primeira infância: relato de caso. Revista Odontológica de Araçatuba, 2011; 32(2): 49-53.

6. CORREIA SF, et al. Defeitos do esmalte: etiologia, características clínicas e diagnóstico diferencial; Revista Instituto de Ciências da Saúde 2007; 25(2):187-92.

7. DE CASTRO JUNIOR, FM. Cirurgia de Cabeça e Pescoço: Tópicos Essenciais. Thieme Revinter, 2019.

8. FAIS L, et al. A idade influencia na satisfação de pacientes usuários de próteses totais. RFO, 2007; $12(2)$ : 37-41.

9. FERREIRA MC, et al. Impact of periodontal disease on quality of life: a systematic review. Periodontal Res, 2017; 52(4):651-665.

10. GALLÃO S, et al. Impacto estético da proporção dentária anterior. Revista Instituto Ciência Saúde, 2009; 27(3): 287-9.

11. KRAMER PF, et al. Traumatismo na dentição decídua e fatores associados em pré-escolares do município de Canela/RS. Pesquisa Brasileira em Odontopediatria e Clínica Integrada, 2009; 9(1): 95-100.

12. LUNARDELLI SE, et al. Autoestima e cárie dentária em adolescentes: um estudo seccional. Revista de Odontologia da UNESP, 2016; 45(6): 332-338.

13. MARCONDES R, et al. Lâmina cerâmica unitária sobre substrato escurecido: protocolo clínico laboratorial com estratificação em duas camadas. Revista Dental Press de Ortodontia e Ortopedia Facial, 2012; 9(3): 28-44.

14. NICODEMO D, et al. Cirurgia ortognática: abordagem psicossocial em pacientes Classe III de Angle submetidos à correção cirúrgica da deformidade dentofacial. Revista Dental Press de Ortodontia e Ortopedia Facial, 2007; 12(5): 45-54.

15. OLIVEIRA D, et al. Restabelecimento estético e funcional de pacientes com amelogênese imperfeita utilizando restaurações cerâmicas metal-free. Arch Health Invest, 2019; 11(7): 465-9.

16. PEDRON IG. Cuidados no planejamento para a aplicação da toxina botulínica em sorriso gengival. Revista de Odontologia da Universidade Cidade de São Paulo, 2017; 26(3):250-256.

17. PINO YC, et al. Tomografía axial computarizada em los traumatismos craneo encefálicos. Hospital Lenin: junio 2015-junio 2016, Holguín, Cuba. Correo Científico Médico, 2020; 24(2).

18. SOUZA AB, et al. A obesidade como fator de risco para doença periodontal: revisão de literatura. Revista Dental Press Periodontia Implantologia, 2010; 4(4):30-9.

19. TIVERON AHB. A importância da correção do sorriso gengival no programa saúde da família. [Trabalho de conclusão de curso]. Uberaba: Universidade Federal de Minas Gerais; 2014.

20. VIEIRA AC, et al. Abordagem Interdisciplinar na reabilitação estética do sorriso. Revista Odontológica de Araçatuba, 2018; 39(2): 54-59. 